\title{
General Psychiatry
}

\section{Regional homogeneity of intrinsic brain activity related to the main alexithymia dimensions}

Dai Han, ${ }^{1,2,3}$ Mei Li, ${ }^{4}$ Minjun Mei, ${ }^{4}$ Xiaofei Sun ${ }^{4}$

To cite: Han D, Li M, Mei M, et al.

Regional homogeneity of intrinsic brain activity related to the main alexithymia dimensions. General Psychiatry 2018;31:e00003. doi:10.1136/ gpsych-2018-000003

Received 05 July 2017 Revised 14 July 2017 Accepted 05 September 2017
Check for updates

(C) Author(s) (or their employer(s)) 2018 . Re-use permitted under CC BY-NC. No commercial re-use. See rights and permissions. Published by BMJ.

${ }^{1}$ Institute of Psychological Sciences, Hangzhou Normal University, Hangzhou, China

${ }^{2}$ Zhejiang Key Laboratory for Research in Assessment of Cognitive Impairments, Hangzhou, China

${ }^{3}$ Center for Cognition and Brain Disorders, Hangzhou Normal University, Hangzhou, China

${ }^{4}$ Mental Health Education and Counseling Center, Hangzhou Normal University, Hangzhou, China

Correspondence to Dai Han, Institutes of Psychological Science, Hangzhou Normal University, Hangzhou 311121, China; handai@hznu.edu.cn

\section{ABSTRACT}

Background Alexithymia is a multidimensional personality construct.

Objective This study aims to investigate the neuronal correlates of each alexithymia dimension by examining the regional homogeneity ( $\mathrm{ReHo}$ ) of intrinsic brain activity in a resting situation.

Methods From university freshmen, students with alexithymia and non-alexithymia were recruited. Their alexithymic traits were assessed using the Toronto Alexithymia Scale-20. The ReHo was examined using a resting-state functional MRI approach.

Results This study suggests significant group differences in $\mathrm{ReHo}$ in multiple brain regions distributed in the frontal lobe, parietal lobe, temporal lobe, occipital lobe and insular cortex. However, only the ReHo in the insula was positively associated with difficulty identifying feelings, a main dimension of alexithymia. The ReHo in the lingual gyrus, precentral gyrus and postcentral gyrus was positively associated with difficulty describing feelings in participants with alexithymia. Lastly, the ReHo in the right dorsomedial prefrontal cortex (DMPFC_R) was negatively related to the externally oriented thinking style of participants with alexithymia.

Conclusion In conclusion, these results suggest that the main dimensions of alexithymia are correlated with specific brain regions' function, and the role of the insula, lingual gyrus, precentral gyrus, postcentral gyrus and DMPFC_R in the neuropathology of alexithymia should be further investigated.

\section{INTRODUCTION}

Alexithymia is a personality trait characterised by poor ability to recognise and describe internal emotional feelings and an externally oriented cognitive style of thinking. ${ }^{1}$ The prevalence of alexithymia has been about $10 \%$ in the general population, but it can be higher in the population with mental disorders such as depression, anxiety, substance misuse, and autism. ${ }^{2-5}$ Alexithymia has also been related to somatic illnesses, including essential hypertension, diabetes mellitus, and psoriasis. ${ }^{6-8}$ Furthermore, a great deal of epidemiological surveys suggest that alexithymia is an unfavourable characteristic for disease control and health promotion. ${ }^{9}$

In recent years, an increasing number of brain imaging studies have been conducted to explore the neurobiological mechanism of alexithymia. Neuroimaging data supported that a high degree of alexithymia was associated with the dysfunction in specific brain regions, such as the medial prefrontal cortex, the anterior cingulate cortex, the insula and so forth. ${ }^{10-12}$ Unfortunately, data on these brain regions involved in alexithymia are still vague, and there are even many self-contradicting research results on the neurobiological models of alexithymia. ${ }^{1314}$ This can be explained by several reasons.

First, alexithymia is a multifaceted personality construct. The main three dimensions of alexithymic personality features are traditionally assessed with the Toronto Alexithymia Scale (TAS-20), which comprises three corresponding subscales: 'difficulty identifying feelings (DIF)', 'difficulty describing feelings (DDF)' and 'externally oriented cognitive style of thinking (EOT)'. Various alexithymic personality features are likely to be linked to different brain regions. ${ }^{15}$ To date, only a few investigators focus on the neural substrates of each dimension of alexithymic personality features. Second, the strong relationship among alexithymia, depression and anxiety has been verified by many investigators. ${ }^{3}{ }^{15}$ Subjects suffering from depression or anxiety should be excluded to reduce disturbance from depression or anxiety symptoms when locating the specific brain structures underlying the essentials of alexithymia. Lastly, most functional imaging studies explored the neurobiological models of alexithymia by observing certain brain regions' activity when subjects are performing a certain task, such as watching affective pictures and facial emotion recognition. Various tasks induced 


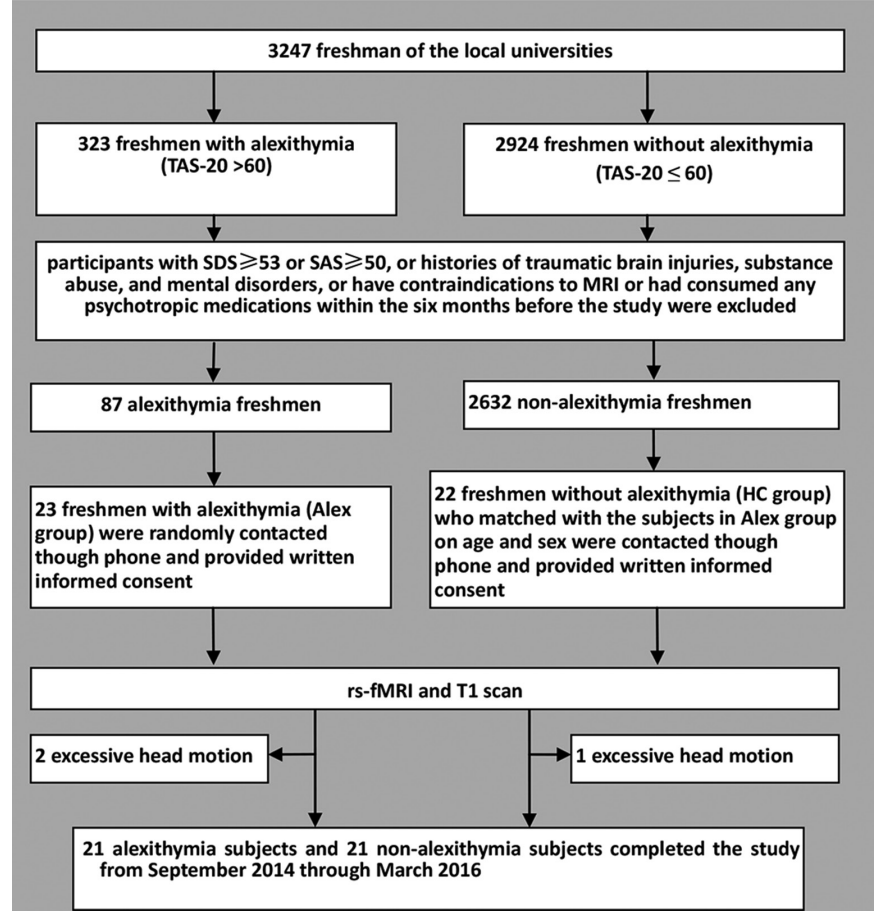

Figure 1 Enrolment of cases and controls.

very different responses in specific brain regions. This can partly explain the inconsistent results of studies on the neural mechanism of alexithymia. Currently, resting-state functional magnetic resonance imaging (rs-fMRI) is increasingly adopted as a natural measurement for brain function because it reflects intrinsic brain interactions at the baseline state, which may provide knowledge on the overall brain function without being biased by differences in task performance during the scanning. ${ }^{16-20}$ The regional homogeneity ( $\mathrm{ReHo}$ ) is an rs-fMRI method that measures the similarity of the time series of a given voxel to those of its nearest neighbours in a voxel-wise way based on the assumption that if a brain region is responsible for a specific function, the voxels within this region were more temporally homogeneous when involved in that function. ReHo is an ideal method to reveal the complexity of the human brain function and is currently one of the most commonly used methods to measure the neural activity in the brain regions. ${ }^{21}$

For these reasons, this study focused on the neural activity underlying the three main dimensions of alexithymic personality features. Furthermore, in this study, participants with depression or anxiety were excluded. To avoid the disturbance from various tasks, this study adapted the ReHo method to investigate the neural functional activity during resting state in the general population with alexithymia.

\section{METHODS}

\section{Subjects}

All of the participants in this study were recruited from the freshmen of local universities, who took a mental health exam as part of the university entrance examination. The participants have to fulfil the following inclusion criteria: right-handed and physically healthy individuals without history of traumatic brain injuries, substance abuse or mental disorders. Participants were excluded if they have contraindications to MRI or had consumed any psychotropic medications within the 6 months before the study. Eligible subjects were randomly contacted through phone, provided written informed consent and effectively completed all of the psychological exams (see figure 1).

According to the results of the psychological measurements (see Psychological measurements section, TAS-20 $>60$, SDS $<53$ and $\mathrm{SAS}<50$ ), 45 participants were recruited from local universities. The data of three participants were excluded from further analysis due to excessive head motion. There were 21 participants in the alexithymia group (Alex group) and 21 participants in the healthy control group (HC group).

\section{Psychological measurements}

The status of alexithymia, depression and anxiety of these participants was assessed, respectively, by the Chinese version of the TAS-20, Self-Rating Depression Scale (SDS) and Self-Rating Anxiety Scale (SAS).

TAS-20 is a 20-item self-report scale graded from 1 (strongly disagree) to 5 (strongly agree). Higher TAS-20 scores indicate a higher level of alexithymia. TAS-20 consists of three subscales: DIF, DDF and EOT. According to the recommendation of the developers of this scale, a TAS-20 total score $>60$ is clinically defined as alexithymia cases. $^{22}$ Therefore, participants with a TAS-20 total score $>60$ were assigned to the Alex group and participants with a TAS-20 total score $\leq 60$ were assigned to the HC group.

SDS and SAS both include 20 items, each graded from 0 to 3 . Higher SDS/SAS scores are associated with more severe depression/anxiety. A gross score on the SDS/SAS is obtained by adding the scores of the 20 questions, and the standardised score is obtained using the integral part of 1.25-fold of the gross score. Subjective depression was defined as a standard SDS score $\geq 53$. Subjective anxiety was defined as an SAS score $\geq 50$. Therefore, all participants' SDS scores must be lower than 53, while the SAS score must be lower than 50 .

\section{MRI measurements}

All MRI measurements were performed with a 3T wholebody magnetic resonance imager (GE MR750) using a standard head coil. Foam padding was used for all subjects to minimise head motion. First, resting-state functional images were acquired (sequence=GREEPI, axial slices, scanning order= interleaved (1:2:43 2:2:42), slice number $=43$, matrix size $=64 \times 64, \mathrm{FOV}=220 \times 220 \mathrm{~mm}$, $\mathrm{TR} / \mathrm{TE}=2000 / 30 \mathrm{~ms}, \mathrm{FA}=90^{\circ}$, slice thickness $=3.2 \mathrm{~mm}$, gap $=0$ (voxel size $3.4 \times 3.4 \times 3.2)$, dummy scan $=0$, number of acquisitions $=240, \mathrm{NEX}=1$, parallel acceleration $=2$, total scan time $=8$ ' 00 "). The subjects were instructed to rest with their eyes closed, not to think of anything in 
particular and not to fall asleep. For structural imaging, high-resolution three-dimensional T1-weighted anatomical images were acquired (sequence $=$ SPGR, parallel acceleration $=2$, sagittal slices, slice number $=180$ with 2 slices in each end discarded (oversampling) to achieve 176 , matrix size $=256 \times 256, \mathrm{FOV}=256 \times 256 \mathrm{~mm}$, TR $/ \mathrm{TE}=$ $8100 / 3.1 \mathrm{~ms}, \mathrm{FA}=8^{\circ}$, slice thickness $=1$, gap $=0$ (isotropic voxel size $=1 \times 1 \times 1$ ), TI (prepare time) $=450 \mathrm{~ms}$, bandwidth $=31.25 \mathrm{kHz}$, total scan time $\left.=5^{\prime} 05^{\prime \prime}\right)$.

\section{Data processing}

Based on Matlab V.2012a (MathWorks, Natick, Massachusetts, USA), data preprocessing was performed using DPARSF (Data Processing Assistant for Resting-State fMRI; http://rfmri.org/DPARSF) and REST (Resting-State fMRI Data Analysis Toolkit; http://www.restfmri.net). ${ }^{23}$ The first 10 images were excluded to ensure stability in the magnetic field. The remaining images were then corrected for slice-time differences and realigned for head motion correction. Data from subjects whose head motion exceeded $1 \mathrm{~mm}$ or for whom rotation exceeded $1^{\circ}$ during scanning were excluded. We then coregistered the individual T1 images to functional images. Normalised data were resliced at a resolution of $3 \times 3 \times 3 \mathrm{~mm}^{3}$ and temporal band-pass filtering $(0.01<\mathrm{f}<0.08 \mathrm{~Hz})$. Functional images with linear trends were removed. Several sources of spurious variance (six head motion parameters, averaged signals from white matter, cerebrospinal fluid and global signals) were regressed out. The ReHo map of each individual was generated using DPARSF. The standardisation of ReHo was done by dividing the ReHo value in each voxel by the mean ReHo values of the whole grey matter. Then the standardised ReHo maps were smoothed with a $6 \mathrm{~mm}$ full width at half-maximum Gaussian kernel to reduce noise.

\section{Statistical analysis}

The demographic data (except for gender) and TAS-20 scores of the Alex group and the HC group were compared by two-sample t-tests, which were conducted using SPSS
V.19.0 statistical software and Excel. Differences were considered significant at a two-sided $p$ value of 0.05 or highly significant at a $\mathrm{p}$ value of 0.01 .

The voxel-by-voxel-based ReHo comparisons between the Alex group and the HC group were performed with two-sample t-test using the REST software $(p<0.01$, AlphaSim correction, cluster size $>20$ ) without age and gender being regressed out because the participants of the two groups were close in age and gender ratio table 1).

The Pearson's correlation between the ReHo values extracted from the brain regions showing significant group differences and the three TAS-20 subscales' scores within the Alex group was calculated $(\mathrm{p}<0.01)$. The analysis was conducted using the SPSS V.19.0 statistical software. Differences were considered significant at a two-sided $p$ value of 0.05 or highly significant at a two-sided $p$ value of 0.01 .

\section{RESULTS}

\section{Demographic and neuropsychological measurement}

The demographic variables and TAS-20 global and three subscales' scores (TAS-DIF, TAS-DDF, TAS-EOT) are presented in table 1 . There were no differences between the Alex group and the HC group in terms of age or gender ratio. The TAS-20 total scores and three subscales' scores in the Alex group were all significantly higher than those in the HC group.

\section{Comparison analysis between the two groups}

The results of the two-sample t-test revealed significant differences in the ReHo between the Alex and HC groups in several regions (figure 2 and table 2). Compared with the HC group, the ReHo values in the ventromedial prefrontal cortex (VMPFC), right dorsomedial prefrontal cortex (DMPFC_R), right middle frontal gyrus (MFG_R), bilateral temporal gyrus (ITG_L\&R), right supramarginal gyrus (SMG_R), and precuneus were significantly higher in the Alex group. In contrast, the ReHo values

Table 1 Comparison of the demographic variables and psychological measurements between the Alex group and the HC group

\begin{tabular}{lllll}
\hline Variables & $\begin{array}{l}\text { Alex group } \\
\mathbf{n = 2 1}\end{array}$ & $\begin{array}{l}\text { HC group } \\
\mathbf{n = 2 1}\end{array}$ & $\mathbf{t}$ & P values \\
\hline $\begin{array}{l}\text { Demographic data } \\
\text { Male, } \mathrm{n}(\%)\end{array}$ & $11(52.4)$ & $11(52.4)$ & & \\
\hline Age (years) & $18.14(1.06)$ & $18.67(1.11)$ & -1.56 & 0.39 \\
\hline TAS-20 scores & & & & \\
TAS total & $63.00(3.22)$ & $41.00(7.75)$ & 11.93 & $<0.001$ \\
TAS-DIF & $24.38(2.92)$ & $13.48(3.52)$ & 10.49 & $<0.001$ \\
\hline TAS-DDF & $17.43(2.23)$ & $10.71(2.57)$ & 9.05 & $<0.001$ \\
\hline TAS-EOT & $21.19(2.96)$ & $16.81(3.87)$ & 4.12 & $<0.001$ \\
\hline
\end{tabular}

Data are presented as percentage/ratio or mean (SD).

Alex group, alexithymia group; DDF, difficulty describing feelings; DIF, difficulty identifying feelings; EOT, externally oriented cognitive style of thinking; HC group, healthy control group; TAS-20, Toronto Alexithymia Scale. 


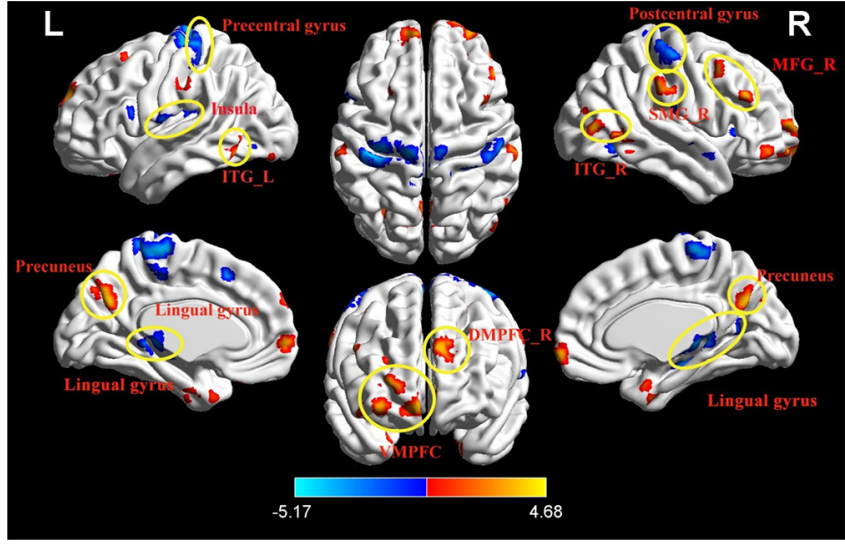

Figure 2 Brain regions showing significant differences in ReHo between the Alex group and the HC group $(\mathrm{p}<$ 0.01, AlphaSim correction, cluster size $>20)$. Alex group, alexithymia group; DMPFC_R, right dorsomedial prefrontal cortex; HC group, healthy control group; ITG_L or R, left or right inferior temporal gyrus; MFG_R, right middle frontal gyrus; ReHo, regional homogeneity; SMG_R, right supramarginal gyrus; VMPFC, ventromedial prefrontal cortex.

in the insula, lingual gyrus, precentral gyrus, and postcentral gyrus were significantly lower in the Alex group compared with the HC group.

\section{Correlation analysis in the Alex group}

The results of the correlation analysis suggested that the TAS-DIF scores were positively associated with the ReHo in the insula $(r=0.52, p=0.016)$ (table 3$)$. The TAS-DDF score was positively and significantly associated with the ReHo in the lingual gyrus $(r=0.45, p=0.043)$, precentral

Table 2 Brain regions showing significant differences in ReHo between the Alex group and the HC group

\begin{tabular}{|c|c|c|c|c|c|}
\hline \multirow[b]{2}{*}{ Brain regions (BA) } & \multirow{2}{*}{$\begin{array}{l}\text { Voxel } \\
\text { size }\end{array}$} & \multicolumn{3}{|c|}{$\begin{array}{l}\text { Peak MNI } \\
\text { coordinate }\end{array}$} & \multirow{2}{*}{$\begin{array}{l}\text { Peak } \\
\text { t-value }\end{array}$} \\
\hline & & $\mathbf{x}$ & $\mathbf{y}$ & $\mathbf{z}$ & \\
\hline VMPFC (47) & 42 & -9 & 63 & 33 & 3.8243 \\
\hline DMPFC_R (10) & 40 & 30 & 66 & 9 & 3.5352 \\
\hline MFG_R (6) & 27 & 30 & 57 & -9 & 3.3345 \\
\hline ITG_L (37) & 164 & -54 & -63 & -12 & 4.6828 \\
\hline ITG_R (37) & 27 & 54 & -78 & 6 & 4.0308 \\
\hline SMG_R (40) & 24 & 66 & -33 & 39 & 3.1414 \\
\hline Precuneus (7) & 27 & 0 & -66 & 30 & 4.0889 \\
\hline Insula (13) & 25 & -30 & -27 & 21 & -3.6762 \\
\hline Lingual gyrus (18) & 153 & -6 & -33 & -6 & -4.2079 \\
\hline Precentral gyrus (4) & 49 & -39 & -27 & 69 & -5.1683 \\
\hline Postcentral gyrus (3) & 95 & 48 & -24 & 57 & -3.5790 \\
\hline
\end{tabular}

$\mathrm{P}<0.01$, AlphaSim correction, cluster size $>19$.

Alex group, alexithymia group; BA, Brodmann area; DMPFC_R, right dorsomedial prefrontal cortex; $\mathrm{HC}$, healthy control group; ITG_L or R, left or right inferior temporal gyrus; MFG_R, right middle frontal gyrus; MNI, Montreal Neurological Institute; ReHo, regional homogeneity; SMG_R, right supramarginal gyrus; VMPFC, ventromedial prefrontal cortex. gyrus $(\mathrm{r}=0.73, \mathrm{p}=0.002)$ and postcentral gyrus $(\mathrm{r}=0.45$, $\mathrm{p}=0.040$ ) (table 3 ). For the TAS-EOT score, the results indicated that the TAS-EOT scores were negatively correlated with the ReHo in the DMPFC_R ( $r=-0.53, \mathrm{p}=$ 0.014) (table 3).

\section{DISCUSSION}

\section{Main findings}

The present study examined the neuronal activity synchronisation in various brain regions of participants with alexithymia during a resting baseline. ReHo reflects the changes in the temporal feature of intrinsic neuronal activity. $^{21}$ The comparative analysis indicated that significant group differences in ReHo were observed within multiple brain regions in the frontal lobe, parietal lobe, temporal lobe, occipital lobe, and insular cortex, including VMPFC, DMPFC_R located in the prefrontal lobe; MFG_R and precentral gyrus located in the frontal lobe; bilateral ITG located in the temporal lobe; SMG_R, precuneus and postcentral gyrus located in the parietal lobe; insula; and the lingual gyrus located in the occipital lobe. Most of these brain regions play an important role in perception, motor control, self-awareness, cognitive function, emotion experience and expression, suggesting the pathology of alexithymia involved in many brain functions. It explains why alexithymia is a multifaceted personality construct.

It is notable that alexithymia is often accompanied by depression and anxiety, and by other mental disorders. These comorbidities confound the symptoms of alexithymia. Consequently, the significant group differences in ReHo were examined in many brain regions, which may not be associated with the development of alexithymia and might be involved in the comorbidities of alexithymia. Therefore, the present study's correlation analysis indicates that only DMPFC_R, insula, lingual gyrus, precentral gyrus and postcentral gyrus are associated with the three dimensions of alexithymia.

The present study suggests that abnormal activity in the insula was positively associated with the TAS-DIF level in individuals with alexithymia. The insula (also called the insular cortex) is a portion of the cerebral cortex folded deep within the lateral sulcus. The insula is commonly believed to be involved in emotions. Additionally, the insula is linked to the functions of interoceptive awareness, self-awareness and social emotions, which include interpersonal experience and empathy processing. ${ }^{24-27}$ The TAS-DIF level indicates the capacity for internal emotional feeling identification, which involves interoceptive awareness, self-awareness, and emotional processing. Previous neuroimaging research had supported that a high degree of alexithymia was associated with a dysfunction in the insula. ${ }^{10}$ This study demonstrates the important role of the insula in the neuropathology of deficits in identifying feelings seen in alexithymia.

In the present study, the TAS-DDF score of individuals with alexithymia was positively associated with the ReHo in the lingual gyrus, precentral gyrus and postcentral gyrus. The TAS-DDF level presents the capacity for describing internal 


\begin{tabular}{|c|c|c|c|c|c|c|}
\hline & \multicolumn{2}{|l|}{ TAS-DIF } & \multicolumn{2}{|l|}{ TAS-DDF } & \multicolumn{2}{|l|}{ TAS-EOT } \\
\hline & $\begin{array}{l}\text { Pearson's } \\
\text { correlation }\end{array}$ & $\begin{array}{l}\text { Significance } \\
\text { (two-tailed) }\end{array}$ & $\begin{array}{l}\text { Pearson's } \\
\text { correlation }\end{array}$ & $\begin{array}{l}\text { Significance } \\
\text { (two-tailed) }\end{array}$ & $\begin{array}{l}\text { Pearson's } \\
\text { correlation }\end{array}$ & $\begin{array}{l}\text { Significance (two- } \\
\text { tailed) }\end{array}$ \\
\hline VMPFC (47) & -0.392 & 0.079 & -0.342 & 0.130 & -0.218 & 0.342 \\
\hline DMPFC_R (10) & 0.184 & 0.424 & -0.177 & 0.443 & $-0.530^{\star}$ & 0.014 \\
\hline MFG_R (6) & -0.263 & 0.250 & -0.195 & 0.398 & -0.157 & 0.497 \\
\hline ITG_L (37) & 0.026 & 0.911 & -0.138 & 0.552 & -0.007 & 0.977 \\
\hline ITG_R (37) & -0.272 & 0.234 & 0.123 & 0.595 & 0.099 & 0.670 \\
\hline SMG_R (40) & -0.027 & 0.906 & 0.139 & 0.547 & 0.080 & 0.730 \\
\hline Precuneus (7) & -0.384 & 0.086 & -0.264 & 0.247 & -0.034 & 0.883 \\
\hline Insula (13) & $0.517^{\star}$ & 0.016 & 0.251 & 0.273 & -0.145 & 0.532 \\
\hline Lingual gyrus (18) & 0.335 & 0.138 & $0.445^{\star}$ & 0.043 & -0.074 & 0.750 \\
\hline Precentral gyrus (4) & 0.140 & 0.546 & $0.731 \dagger$ & 0.002 & -0.035 & 0.840 \\
\hline Postcentral gyrus (3) & 0.128 & 0.581 & $0.451^{*}$ & 0.040 & 0.047 & 0.879 \\
\hline
\end{tabular}

${ }^{*}$ Correlation is significant at the 0.05 level.

†Correlation is significant at the 0.01 level.

Alex group, alexithymia group; DDF, difficulty describing feelings; DIF, difficulty identifying feelings; DMPFC_R, right dorsomedial prefrontal cortex; EOT, externally oriented cognitive style of thinking; ITG_L or R,left or right inferior temporal gyrus; MFG_R, right middle frontal gyrus; ReHo, regional homogeneity; TAS-20, Toronto Alexithymia Scale; SMG_R, right supramarginal gyrus; VMPFC, ventromedial prefrontal cortex.

emotional feelings. The precentral gyrus is located in the dorsal portion of the frontal lobe. It is the primary region of the motor system which plans, controls and executes voluntary movements. Animal research suggested that the precentral gyrus may participate in integrating muscles in meaningful ways rather than in segregating the control of individual muscle groups. ${ }^{28}$ The lingual gyrus is located in the bottom part of the occipital lobe. Lingual gyrus activation has been linked to processing visual information and encoding visual memories. ${ }^{29}{ }^{30}$ The postcentral gyrus is a part of the parietal lobe and behind the precentral gyrus. It is the main sensory receptive area for somatosensory information. Suslow and colleagues ${ }^{31}$ suggested that the somatosensory cortices were known to be critically involved in the processes of emotional mimicry. Humans express emotional feelings mainly through speaking, facial expression or body movement. Previous research had supported that facial somatosensory/motor activity can play a role in how faces are processed. ${ }^{32}$ Therefore, it is reasonable to infer that the TAS-DDF levels are involved in the lingual gyrus, precentral gyrus and postcentral gyrus.

For the TAS-EOT level in participants with alexithymia, the results of the present study indicated that ReHo in the right DMPFC was negatively associated with alexithymia. DMPFC is located in the mid-dorsolateral part of the prefrontal lobe. Isoda and colleagues ${ }^{33}$ reported that DMPFC was involved in the 'theory of mind' or considering the mental states of other peoples. In recent years, many studies have emphasised the relationship between alexithymia and the "theory of mind'. ${ }^{34}$ The TAS-EOT level presents the externally oriented cognitive style of thinking in people with alexithymia. Additionally, DMPFC has been demonstrated to play an important role in various complex cognitive processes, such as social cognition, acquisition of goal-directed behaviour, devaluation of the outcome and resolving conflict. ${ }^{36-38}$ According to these previous reports and the results of the present study, the right DMPFC should play an important role in the development of alexithymia and is especially involved in the EOT of people with alexithymia.

\section{Limitations}

Regretfully, the number of subjects with alexithymia was limited in the present study. In order to better understand the roles of these three brain regions in the development of the three main features of alexithymia, a further investigation recruiting more subjects is necessary.

\section{Implications}

In conclusion, this study examined the neuronal activity in persons with alexithymia and analysed the relationship between the three dimensions of alexithymia and neuronal activity in specific brain regions. This study suggested that the three dimensions of alexithymia were linked to different brain regions (including insula, right DMPFC, lingual gyrus, precentral gyrus and postcentral gyrus) separately. The functional alteration of these brain regions in persons with alexithymia should be further investigated for a more complete understanding of the neuronal mechanism underlying alexithymia.

Contributors HD designed the study, managed the imaging data selection and analysis, and wrote the first draft of the manuscript. LM, MM and SX undertook the psychological measurements and statistical analysis. All authors contributed to and have approved the final manuscript.

Funding This work was supported by the Natural Science Foundation of Zhejiang Province (no Q14H090014).

Competing interests None declared.

Patient consent Obtained 
Provenance and peer review Not commissioned, externally peer reviewed.

Data statement No additional data are available.

Open access This is an open access article distributed in accordance with the Creative Commons Attribution Non Commercial (CC BY-NC 4.0) license, which permits others to distribute, remix, adapt, build upon this work non-commercially, and license their derivative works on different terms, provided the original work is properly cited and the use is non-commercial. See: http://creativecommons.org/ licenses/by-nc/4.0

\section{REFERENCES}

1. Bagby RM, Parker JD, Taylor GJ. Disorders of affect regulation: Alexithymia in medical and psychiatric illness. Cambridge, UK: Cambridge University Press, 1997.

2. Karukivi M, Saarijärvi S. Development of alexithymic personality features. World J Psychiatry 2014;4:91-102.

3. Honkalampi K, Koivumaa-Honkanen $\mathrm{H}$, Lehto $\mathrm{SM}$, et al. Is alexithymia a risk factor for major depression, personality disorder, or alcohol use disorders? A prospective population-based study. $J$ Psychosom Res 2010;68:269-73.

4. Allen LB, Qian Lu L, Tsao JC, et al. Depression partially mediates the relationship between alexithymia and somatization in a sample of healthy children. J Health Psychol 2011;16:1177-86.

5. Cook R, Brewer R, Shah P, et al. Alexithymia, not autism, predicts poor recognition of emotional facial expressions. Psychol Sci 2013;24:723-32.

6. Grabe HJ, Schwahn C, Barnow S, et al. Alexithymia, hypertension, and subclinical atherosclerosis in the general population. $J$ Psychosom Res 2010;68:139-47.

7. Chatzi L, Bitsios P, Solidaki E, et al. Type 1 diabetes is associated with alexithymia in nondepressed, non-mentally ill diabetic patients: a case-control study. J Psychosom Res 2009;67:307-13.

8. Richards HL, Fortune DG, Griffiths CE, et al. Alexithymia in patients with psoriasis: clinical correlates and psychometric properties of the Toronto Alexithymia Scale-20. J Psychosom Res 2005;58:89-96.

9. Kojima M. Alexithymia as a prognostic risk factor for health problems: a brief review of epidemiological studies. Biopsychosoc Med 2012;6:21.

10. Jongen S, Axmacher N, Kremers NA, et al. An investigation of facial emotion recognition impairments in alexithymia and its neural correlates. Behav Brain Res 2014;271:129-39.

11. Reker M, Ohrmann P, Rauch AV, et al. Individual differences in alexithymia and brain response to masked emotion faces. Cortex 2010;46:658-67.

12. Pouga L, Berthoz S, de Gelder B, et al. Individual differences in socioaffective skills influence the neural bases of fear processing: the case of alexithymia. Hum Brain Mapp 2010;31:1469-81.

13. Mantani T, Okamoto $Y$, Shirao N, et al. Reduced activation of posterior cingulate cortex during imagery in subjects with high degrees of alexithymia: a functional magnetic resonance imaging study. Biol Psychiatry 2005;57:982-90.

14. Kano M, Fukudo S, Gyoba J, et al. Specific brain processing of facial expressions in people with alexithymia: an H2 15O-PET study. Brain 2003;126(Pt 6):1474-84.

15. Hendryx MS, Haviland MG, Shaw DG. Dimensions of alexithymia and their relationships to anxiety and depression. $J$ Pers Assess 1991;56:227-37.

16. Raichle ME, Gusnard DA. Intrinsic brain activity sets the stage for expression of motivated behavior. J Comp Neurol 2005;493:167-76.
17. Calhoun VD, Adali T, Pearlson GD, et al. A method for making group inferences from functional MRI data using independent component analysis. Hum Brain Mapp 2001;14:140-51.

18. van de Ven VG, Formisano E, Prvulovic D, et al. Functional connectivity as revealed by spatial independent component analysis of fMRI measurements during rest. Hum Brain Mapp 2004;22:165-78.

19. Fox MD, Raichle ME. Spontaneous fluctuations in brain activity observed with functional magnetic resonance imaging. Nat Rev Neurosci 2007;8:700-11.

20. Biswal B, Yetkin FZ, Haughton VM, et al. Functional connectivity in the motor cortex of resting human brain using echo-planar MRI. Magn Reson Med 1995;34:537-41.

21. Zang $\mathrm{Y}$, Jiang $\mathrm{T}$, Lu Y, et al. Regional homogeneity approach to fMRI data analysis. Neuroimage 2004;22:394-400.

22. Bagby RM, Parker JD, Taylor GJ. The twenty-item Toronto alexithymia scale--I. Item selection and cross-validation of the factor structure. J Psychosom Res 1994;38:23-32.

23. Song XW, Dong ZY, Long XY, et al. REST: a toolkit for resting-state functional magnetic resonance imaging data processing. PLoS One 2011;6:e25031

24. Critchley HD, Wiens $S$, Rotshtein P, et al. Neural systems supporting interoceptive awareness. Nat Neurosci 2004;7:189-95.

25. Craig AD, Craig AD. How do you feel--now? The anterior insula and human awareness. Nat Rev Neurosci 2009;10:59-70.

26. Phan KL, Wager T, Taylor SF, et al. Functional neuroanatomy of emotion: a meta-analysis of emotion activation studies in PET and fMRI. Neuroimage 2002;16:331-48.

27. Ortigue S, Grafton ST, Bianchi-Demicheli F. Correlation between insula activation and self-reported quality of orgasm in women. Neuroimage 2007;37:551-60.

28. Graziano MS, Taylor CS, Moore T. Complex movements evoked by microstimulation of precentral cortex. Neuron 2002;34:841-51.

29. Bogousslavsky J, Miklossy J, Deruaz JP, et al. Lingual and fusiform gyri in visual processing: a clinico-pathologic study of superior altitudinal hemianopia. J Neurol Neurosurg Psychiatry 1987;50:607-14.

30. Kozlovskiy SA, Pyasik MM, Korotkova AV, et al. Activation of left lingual gyrus related to working memory for schematic faces. Int $J$ Psychophysiol 2014;94:241.

31. Suslow T, Kugel H, Rauch AV, et al. Attachment avoidance modulates neural response to masked facial emotion. Hum Brain Mapp 2009;30:3553-62.

32. Wood A, Lupyan G, Sherrin S, et al. Altering sensorimotor feedback disrupts visual discrimination of facial expressions. Psychon Bull Rev 2016;23:1150-6.

33. Isoda M, Noritake A. What makes the dorsomedial frontal cortex active during reading the mental states of others? Front Neurosci 2013;7:232.

34. Brewer R, Happé F, Cook R, et al. Commentary on "Autism, oxytocin and interoception": alexithymia, not autism spectrum disorders, is the consequence of interoceptive failure. Neurosci Biobehav Rev 2015;56:348-53.

35. Demers LA, Koven NS. The relation of alexithymic traits to affective theory of mind. Am J Psychol 2015;128:31-42.

36. Ostlund SB, Balleine BW. Lesions of medial prefrontal cortex disrupt the acquisition but not the expression of goal-directed learning. $J$ Neurosci 2005;25:7763-70.

37. de Wit S, Kosaki Y, Balleine BW, et al. Dorsomedial prefrontal cortex resolves response conflict in rats. J Neurosci 2006;26:5224-9.

38. Eickhoff SB, Laird AR, Fox PT, et al. Functional segregation of the human dorsomedial prefrontal cortex. Cereb Cortex 2016;26:304-21.

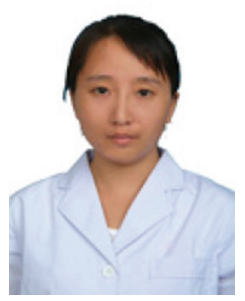

Dai Han obtained a bachelor's degree in clinical medicine from the Medical University of Anhui in 2002, and a PhD in psychiatry and mental health from Fudan University, Shanghai, China in 2013. At present, she is a lecturer in the Institute of Psychological Science at Hangzhou Normal University and a psychiatrist in the Affiliated Hospital of Hangzhou Normal University. Her research interests include clinical neuropsychology, exploring the pathogenesis and psychological intervention mechanism of mental disorders and organic brain diseases. 